\title{
Dynamic filamentation and beam quality of quantum-dot lasers
}

\author{
E. Gehrig a) and O. Hess \\ Advanced Technology Institute, School of Electronics and Physical Sciences, University of Surrey, \\ Guildford, Surrey, GU2 7XH, United Kingdom \\ C. Ribbat, R. L. Sellin, and D. Bimberg \\ Institut für Festkörperphysik, Technische Universität Berlin, D-10623 Berlin, Germany
}

(Received 21 August 2003; accepted 21 January 2004)

\begin{abstract}
We present a comparative study of numerical simulations and experiments on the spatiotemporal dynamics and emission characteristics of quantum-well and quantum-dot lasers of identical structure. They show that, in the quantum-dot laser, the strong localization of carrier inversion and the small amplitude-phase coupling enable a significant improvement of beam quality compared to quantum-well lasers of identical geometry. Near-field profiles and beam quality $\left(M^{2}\right)$ parameters calculated on the basis of time-dependent effective Maxwell-Bloch equations into which the physical properties of the active media are included via space-dependent material parameters, effective time constants, and matrix elements are fully confirmed by experimental measurements. Together they indicate that, in the quantum-dot laser, the strong localization of carrier inversion and the small amplitude-phase coupling enable a significant improvement of beam quality compared with quantum-well lasers of identical geometry. (C) 2004 American Institute of Physics.
\end{abstract}

[DOI: $10.1063 / 1.1668330]$

Impressive technological progress in the field of quantum-dot lasers ${ }^{1}$ that exploit characteristic physical properties of quantum dots has, in recent years, led to the concept and realization of promising laser sources for the generation of long-wavelength radiation with good spatial and spectral purity as required by many applications, such as, for example, optical communication networks. ${ }^{2}$ For many applications, the prospect of realizing innovative high-power quantum-dot lasers with a reduced tendency of detrimental spatiotemporal filamentation dynamics is particularly important. First theoretical and experimental investigations on the linewidth enhancement factor ( $\alpha$-factor) have shown that the quantum-dot laser exhibits less filamentation than the quantum-well laser. ${ }^{3}$ In this letter, we investigate the spatiotemporal dynamics and beam quality of InGaAs quantumdot lasers (dot density $10^{11} \mathrm{~cm}^{-2}$ ) emitting at $1.1 \mu \mathrm{m}$. For direct comparison with quantum-well lasers, we contrast and compare our theoretical and experimental results with corresponding lasers with a quantum-well gain medium, but identical waveguide structure.

Our theoretical analysis is based on an effective multimode Maxwell-Bloch approach ${ }^{4}$ that takes into account, in particular, the spatially and temporally varying material properties (of, e.g., the quantum-well or the active dot ensemble) and physical interactions that have a detrimental influence on beam quality and spectral characteristics. Simulations on the basis of this approach provide an interpretation of experimental results such as near-field profiles, power, and beam quality factor $M^{2} .^{5}$

The multimode Maxwell-Bloch equations consist of multimode wave equations and two-level Bloch equations for the dynamics of carriers as well as the dipole dynamics within the active media. The spatially dependent multimode

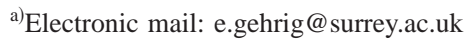

wave equations for the dynamics of the light fields propagating in forward ("+") and backward ("-") direction read

$$
\frac{\partial}{\partial t} E^{ \pm}+\frac{\partial}{\partial z} E^{ \pm}=i D_{p} \frac{\partial^{2}}{\partial x^{2}} E^{ \pm}-i \eta E^{ \pm}+\Gamma P_{(0)}^{ \pm} .
$$

The diffraction coefficient is $D_{p}=\left(2 n_{l} k_{0}\right)^{-1}$ with the vacuum wave number $k_{0}=2 \pi / \lambda$. The waveguiding properties derived from effective index approximation are included in the parameter $\eta$, and $\Gamma$ is the confinement factor. Via the polarization, the light fields are locally coupled to carriers within the active medium. On the basis of an effective twolevel description of the material properties of the active medium (e.g., quantum well or quantum dot), the dynamics of the carrier density and the polarization can be described by the following Bloch equations:

$$
\begin{aligned}
\frac{\partial}{\partial t} P_{(0)}^{ \pm}= & -\gamma_{p}\left[\left(1+i \frac{\bar{\omega}}{\gamma_{p}}\right)+(\rho+i \sigma) N\right] P_{(0)}^{ \pm} \\
& +\beta\left(\left(N_{(0)}+i \alpha\right) E^{ \pm}+N_{(1)} E^{\mp}\right) \\
\frac{\partial}{\partial t} P_{(1)}^{ \pm}= & -\gamma_{p}\left[\left(1+i \frac{\bar{\omega}}{\gamma_{p}}\right)+(\rho+i \sigma) N\right] P_{(1)}^{ \pm}+\beta N_{(1)} E^{ \pm} \\
\frac{\partial}{\partial t} N_{(0)}=\Lambda & +D_{f} \nabla^{2} N_{(0)}-\gamma_{\mathrm{nr}} N_{(0)}-2\left(E^{+}\left(P_{(0)}^{+}-\Lambda_{0} E^{+}\right)^{*}\right. \\
+ & \left.E^{-}\left(P_{(0)}^{-}-\Lambda_{0} E^{-}\right)^{*}+\text { c.c. }\right) \\
\frac{\partial}{\partial t} N_{(1)}= & -4 D_{f} k_{z}^{2} N_{(1)}-\gamma_{\mathrm{nr}} N_{(1)}-2\left(E^{+}\left(P_{(0)}^{-}-\Lambda_{0} E^{-}\right)^{*}\right. \\
& \left.+E^{-*}\left(P_{(0)}^{+}-\Lambda_{0} E^{+}\right)+E^{+*} P_{(1)}^{+}+E^{-} P_{(1)}^{-*}\right) .
\end{aligned}
$$

In Eqs. (1) and (2) $P_{(0)}^{ \pm}$, the parameters $P_{(0)}^{ \pm}, P_{(1)}^{ \pm}, N_{(0)}$, and $N_{(1)}$ are the (lowest and first-order) coefficients of the mode expansion. $\Lambda$ describes the carrier injection into the 
stripes, $D_{f}$ is the carrier diffusion constant, and $k_{z}$ denotes the wave number of the propagating light fields. $\bar{\omega}$ is the frequency detuning between the frequency of the electronhole pair and the light frequency. $\gamma_{\mathrm{nr}}$ describes the rate of nonradiative recombination and $\gamma_{p}$ is the dephasing of the dipole. The dimensionless constant $\beta$ determines the maximum gain. The material parameters $\rho$ and $\sigma$ consider the increase in the polarization decay rate and the drift of the gain maximum with increasing carrier density, respectively. Derived from microscopic calculations, ${ }^{4}$ they include the individual material properties of the quantum-well or quantumdot device. The $\alpha$-factor describes the amplitude-phase coupling. The parameter $\Lambda_{0}$ guarantees a vanishing gain at transparency.

Note that Eq. (2) constitutes a very general description of the dipole dynamics within the active layer of a semiconductor laser. Its application to a quantum-dot structure requires some modifications to account for the characteristics of a quantum-dot laser ensemble: First, in order to model the dynamic carrier escape and carrier capture from the wetting layer states, we include in the equation for the carrier density an additional term $\partial N_{0} / \partial t \mid$ describing carrier escape and carrier capture into the dot from the wetting layer. ${ }^{6}$ The dynamics of the carriers in the layers surrounding the dots thereby is described by a diffusion equation. ${ }^{6}$ Secondly, $P_{(0,1)}^{+-}(i, j)$ and $\bar{\omega}$ refer to "interlevel dipoles" and frequencies of the respective electron and hole levels (with level index " $i$ " for electrons and " $j$ " for holes, respectively). Furthermore, the $\alpha$ factor typically has a value of $2.5-3$ in a quantum-well laser whereas the quantum-dot laser is characterized by a reduced amplitude-phase coupling of 1-1.5. The multimode Maxwell-Bloch equations [Eqs. (1) and (2)] taking into account the dynamic interaction of the counterpropagating light fields with spatially dependent microscopic properties of the active media will in the following be applied to quantumwell and quantum-dot lasers of identical waveguide structure.

Figure 1 shows, in direct comparison, the theoretical [Figs. 1(a) and 1(b)] and experimental [Fig. 1(c)] results of the nearfield characteristics of quantum-well Fig. 1 (lefthand side) and quantum-dot lasers Fig. 1 (right-hand side) of identical waveguide design. The width of the lasers is $6 \mu \mathrm{m}$, the cavity length was $1.3 \mathrm{~mm}$. Figure 1(a) shows the calculated near-field dynamics of a quantum-well (left-hand side) and quantum-dot laser (right-hand side). In the example, the injection current density was chosen such that the output power was $60 \mathrm{~mW}$. For small output powers, the near field of a quantum-well laser is still rather uniform. However, if we operate the laser at higher-power levels, a very different behavior can be observed. Physical processes, such as carrier diffusion and scattering in combination with light diffraction, lead to a complex transverse migration of the light fields [Fig. 1(a) left-hand side]. In contrast, the transverse dynamics of the light fields propagating in the quantum-dot laser [Fig. 1(a) right-hand side] is still rather uniform (for the same output power). This complex light field dynamics are governed by the (space- and time-dependent) mutual interplay of carriers with spontaneous and induced emission processes. In combination with (counter-) propagation effects and transverse migration of the light fields, it affects and Downloaded 30 Mar 2009 to 131.227.178.132. Redistribution subje
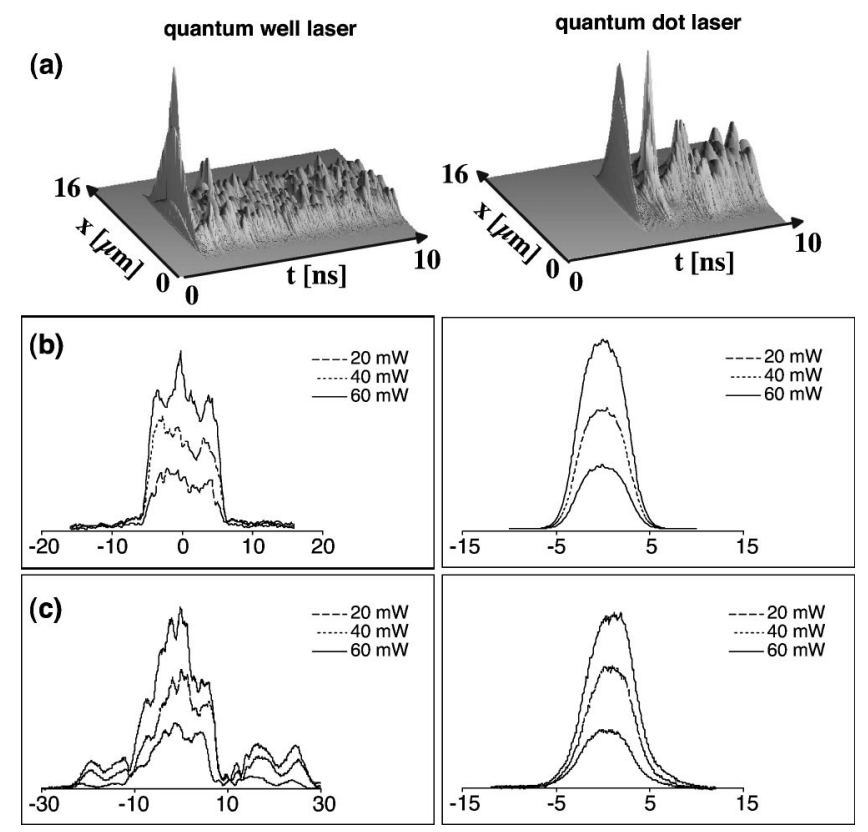

FIG. 1. Simulated spatiotemporal nearfield dynamics (a) and temporally averaged near fields [(b): theory, (c) experiment] at the output facet of a quantum-well (left-hand side) and a quantum-dot (right-hand side) laser. Both lasers have the same geometry (width $10 \mu \mathrm{m}$, cavity length $1 \mathrm{~mm}$ ).

determines the near field and beam quality that can be measured and observed in an experimental investigation.

A comparison of the calculated and measured timeaveraged near fields of a quantum-well laser (left-hand side) and a quantum-dot laser (right-hand side) [Figs. 1(b) and 1(c)] demonstrates that the increased influence of the transverse degree of freedom leads in the quantum-well laser to the formation of filaments whereas the quantum-dot laser shows a Gaussian-shaped uniform near-field distribution. The theoretical results obtained with the Maxwell-Bloch equations are in good agreement with the measured nearfield distributions [Fig. 1(c)]. The side lobes next to the laser ridge that can be seen in Fig. 1(c) result from current spreading in the cladding and waveguide layers. The suppressed transverse light field dynamics observed in experiment and simulation clearly demonstrate the promising device performance of quantum-dot lasers compared to large area lasers and laser amplifiers which show a strong tendency for filamentation formation. ${ }^{7-9}$

By varying the carrier injection current and the laser width in the simulation, we have systematically analyzed the spatiotemporal light field dynamics of quantum-well and quantum-dot lasers. The theoretical and measured values of the beam quality factor $M^{2}$ as obtained by the spatiotemporal simulation are depicted in Fig. 2 in dependence on stripe width [Fig. 2(a)] and output power [Fig. 2(b)] (for the same output power of $20 \mathrm{~mW}$ ). With increasing stripe width [Fig. 2(a)], the transverse degree effectively gets more important: Physical processes, such as carrier diffusion and diffraction of the light fields, lead to characteristic dynamic optical patterns that typically lie in the $\mu \mathrm{m}$ regime. In combination with the dynamic phase changes, this results in a deterioration of the beam quality, i.e., the $M^{2}$ parameter of the quantum-well and the quantum-dot laser increases with increasing stripe to AIP license or copyright; see http://apl.aip.org/apl/copyright.jsp 

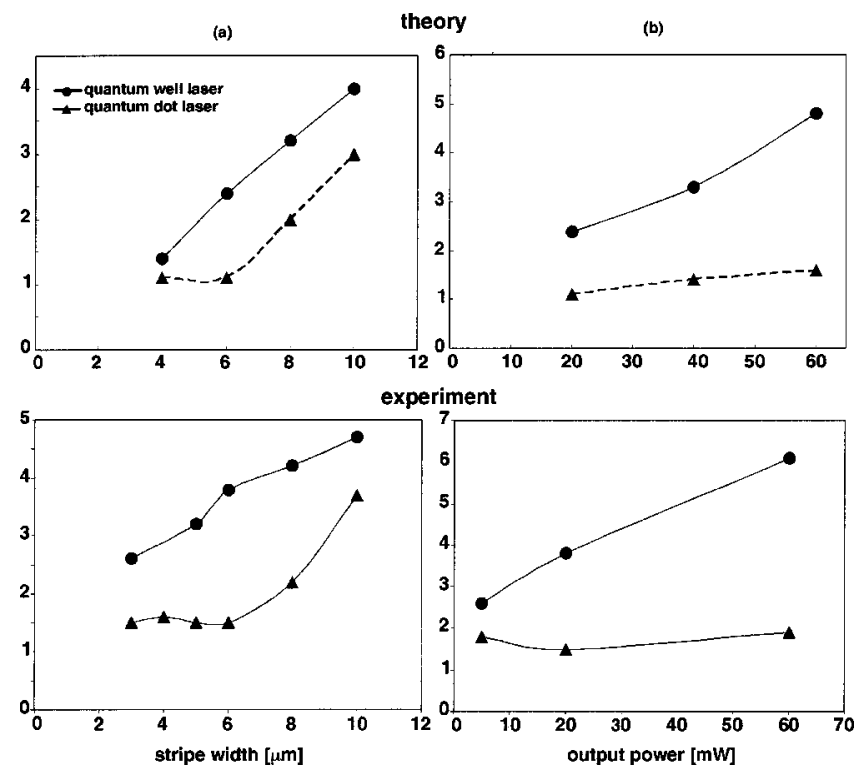

FIG. 2. Beam quality parameter $\left(M^{2}\right)$ in dependence of stripe width (a) and output power (b).

width. However, due to the strong localization of the carriers and the reduced $\alpha$-factor, the $M^{2}$ values of the quantum-dot laser are always smaller then the respective values of the quantum-well structure. In particular, the quantum-dot laser shows a characteristic threshold near $8 \mu \mathrm{m}$. In this intermediate stripe regime, the quantum-dot laser is still single mode whereas $M^{2}>2$ for the quantum-well laser. The dependence of $M^{2}$ on output power is shown in Fig. 2(b) (for a stripe width of $6 \mu \mathrm{m})$. In the quantum-well laser, an increase in the injection current density not only increases the output power but simultaneously leads to an increase in the $M^{2}$ parameter. This is a direct consequence of dynamic carrier diffusion and light diffraction affecting the light fields during their propagation in the laser. In contrast, the quantum-dot laser shows almost no dependence on output power. The dependence of $M^{2}$ on stripe width and output power can be confirmed by the experimental measurements performed on the same devices. Our numerical results clearly demonstrate that quantum-dot lasers have a much better beam quality compared to quantum-well lasers of same geometry. The strong localization of the carriers in the dots in combination with the reduced amplitude phase coupling thus guarantees a good spatial quality.

For an analysis of the spectral properties Fig. 3 shows calculated (spatially resolved) emission spectra of the quantum-well (left-hand side) and the quantum-dot laser (right-hand side). In Fig. 3, the vertical axis denotes the lateral coordinate of the laser and the horizontal axis refers to the frequency. Both structures show a set of longitudinal modes that coexist in the laser-according to the Fabry-Perot modes of the cavity. In the quantum-well laser [Fig. 3(a)], the width of the laser is larger than typical interaction length scales of the laser. As a consequence, a characteristic transverse spatiospectral coupling arises leading to spectral broadening of the individual modes. In addition, each longitudinal

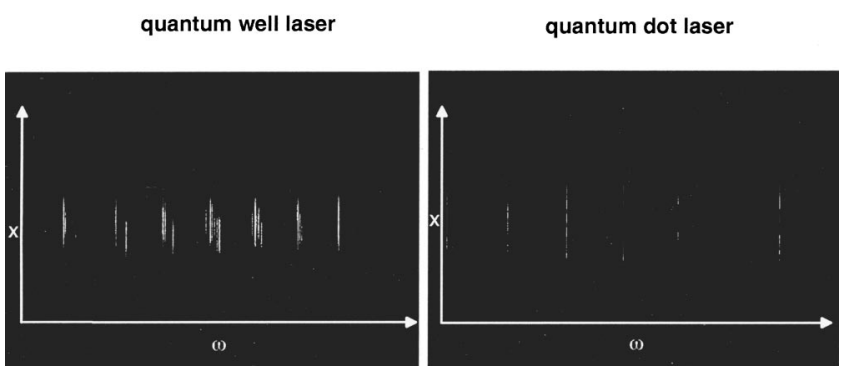

FIG. 3. Calculated emission spectrum of a quantum-well (left-hand side) and a quantum-dot laser (right-hand side).

mode is surrounded by a set of transverse modes. In the case of the quantum-dot laser, the strong carrier localization and the discrete energy levels lead to reduced carrier diffusion, a reduction of transverse dynamics, and low amplitude-phase coupling ( $\alpha$-factor). As a consequence, the emission spectrum is of much higher spectral purity than in the situation of the quantum-well laser.

In conclusion, we have shown numerical and experimental results on the spatiotemporal dynamics and beam quality of quantum-dot and quantum-well lasers. For characteristic sets of material parameters describing the active quantumwell or the quantum-dot media, the effective MaxwellBloch equations allow a realistic simulation of the spatiotemporal dynamics of quantum-dot and quantum-well lasers complementing experimentally measured near-field profiles and beam quality factors. The self-consistent inclusion of all relevant geometrical parameters and material properties (e.g., refractive index and waveguide structure, dot density, and spatial dot distribution) provides a fundamental description of the underlying physical processes and guarantees a realistic modeling of the laser behavior. In particular, the simulations allow the systematic variation of the individual parameters and properties with respect to their influence on beam quality and power. In combination, the theoretical and experimental investigations clearly indicate that the quantumdot laser is a highly promising laser source for the generation of long-wavelength radiation with improved spatial and spectral purity. Experiment and modeling together may thus significantly contribute to the development of optimized innovative quantum-dot devices.

${ }^{1}$ D. Bimberg, M. Grundmann, and N. N. Ledentsov, Quantum Dot Heterostructures (Wiley, Chichester, 1999).

${ }^{2}$ D. Bimberg and N. N. Ledentsov, J. Phys.: Condens. Matter 15, R1 (2003).

${ }^{3}$ P. M. Smowton, E. J. Pearce, H. C. Schneider, W. W. Chow, and M. Hopkonson, Appl. Phys. Lett. 81, 3251 (2002).

${ }^{4}$ E. Gehrig and O. Hess, Spatiotemporal Dynamics and Quantum Fluctuations in Semiconductor Lasers (Springer, Heidelberg, 2003).

${ }^{5}$ C. Ribbat, R. Sellin, I. Kaiander, F. Hopfer, N. N. Ledentsov, D. Bimberg, A. R. Kovsh, V. M. Ustinov, A. E. Zhukov, and M. Maximov, Appl. Phys. Lett. 82, 952 (2003).

${ }^{6}$ E. Gehrig and O. Hess, Phys. Rev. A 65, 033804 (2002).

${ }^{7}$ R. Lang, A. Hardy, R. Parke, D. Mehuys, and S. O'Brien, IEEE J. Quantum Electron. 30, 658 (1994).

${ }^{8}$ J. R. Marciante and G. P. Agrawal, IEEE J. Quantum Electron. 32, 590 (1996).

${ }^{9}$ E. Gehrig, O. Hess, and R. Wallenstein, IEEE J. Quantum Electron. 35, 320 Q331 (1999). 